\title{
Assessment of Knowledge and Practices of Health Care Providers about Neonatal Jaundice in Primary Health Care Units in Fayoum Governorate
}

\author{
Al Kassem Ahmed Al Gameel ${ }^{(1)}$, Naglaa Abd El-Khalek El-Sherbiny ${ }^{(2)}$, Wael Sayed \\ Mohamed $^{(3)}$, Rehab Ewais Hussein Amin ${ }^{(4)}$ \\ 1- Assistant Professor of Pediatrics Faculty of Medicine, Fayoum University. \\ 2- Professor of Public Health \&Community Medicine Faculty of Medicine, \\ Fayoum University. \\ 3- Lecturer of Pediatrics Faculty of Medicine, Fayoum University. \\ 4- (M.B.B.CH), Faculty of Medicine, Fayoum University. \\ Corresponding author: Dr Al Kassem Ahmed Al Gameel,
}

E-mail: a_algameel@icloud.com

Tel: 01001382144

\begin{abstract}
Background: Neonatal jaundice is still a leading cause of preventable brain damage, physical and mental handicap, and early death among infants in many communities. Greater awareness is needed among all health workers.
\end{abstract}

Aim: Assessment of knowledge and practices of primary health care workers about description, causes, effective treatment and complication of neonatal jaundice. Subject and methods: the study was conducted during the period from November, 2017 till October, 2018 on 315 nurses and 152 doctors all were worked in primary health care centers and units at the six districts of Fayoum Governorate it was based on a self-administered, structured, piloted two questionnaires, Arabic one for nurses and an English one for physicians. Both the questionnaires assessed the knowledge and practice of the nurses and physicians regarding neonatal jaundice.
Results: the knowledge score of study physicians regarding neonatal jaundice was poor $(20.4 \pm 3.1$ of total score 42$)$, While the total practices of them were generally good $(14.8 \pm 2.1$ of total score 24$)$. The total knowledge score of nurses regarding neonatal jaundice was poor $(13.8 \pm 4.5$ of total score 32), while the total practice of theme were generally good $(7.6 \pm 1.8$ of total score 12). Conclusion: knowledge of primary health care workers about neonatal jaundice was inadequate and may cause potential delays in referral for effective treatment. There is need for regular training and re-training of primary health care workers to ensure effective management and reduce the complications of neonatal jaundice.

Keywords: Assessment, Knowledge,

Primary health workers, Neonatal jaundice, Fayoum Governorate.

\section{Introduction:}


Neonatal hyperbilirubinaemia is one of the most common disorders in newborn babies, which result from a predisposition of bilirubin in newborn infants and their limited ability to excrete it. Neonatal hyperbilirubinaemia affects approximately 2.4-15\% newborns during the first 2 weeks of life (Li et al., 2019).

In the majority of infants, jaundice resolves spontaneously and causes no harm; however, in some babies, significant hyperbilirubinemia can develop.

Low concentrations of bilirubin are generally harmless and may have some antioxidant benefits, whereas high bilirubin levels may cause bilirubin encephalopathy, even the survivors may develop chronic or permanent damage and sequelae in the central nervous system (Li et al., 2019).

Timely and appropriate treatment with phototherapy and/or exchange transfusion is effective in controlling excessive bilirubin levels in the affected infants. Otherwise, severe hyperbilirubinaemia may progress to acute bilirubin encephalopathy (ABE) or kernicterus with a significant risk of mortality in newborns (Olusanya et al., 2015).

Primary health facilities and the workers are the closest health care providers to the community in terms of availability, accessibility and affordability. The role of primary health care levels is very critical to neonatal jaundice management. Parents heavily depend in most cases on the advice and care being provided by these facilities without cross checking whether they are right or not(Adebami, 2015).

\section{Study Design:}

The current study was a cross sectional study; conducted at Fayoum Governorate primary health care (PHC) centers and units during the period from November, 2017 till October, 2018 to assess the knowledge and practices of the nurses and primary health care physicians about neonatal jaundice.

\section{Statistical Analysis:}

The collected questionnaires were revised for completeness and logical consistency. Recoded data was entered on computer using Microsoft excel software program. Data was then transferred to the Statistical Package for Social Science (SPSS) version 18 to be statistically analyzed by the following levels of analysis.

\section{Results :}

. The majority of study participants $132(86.8 \%)$ lacked any specific training about neonatal jaundice. All of the studied physicians reported their needs in receiving training and information about neonatal jaundice as shown in figures (1). 


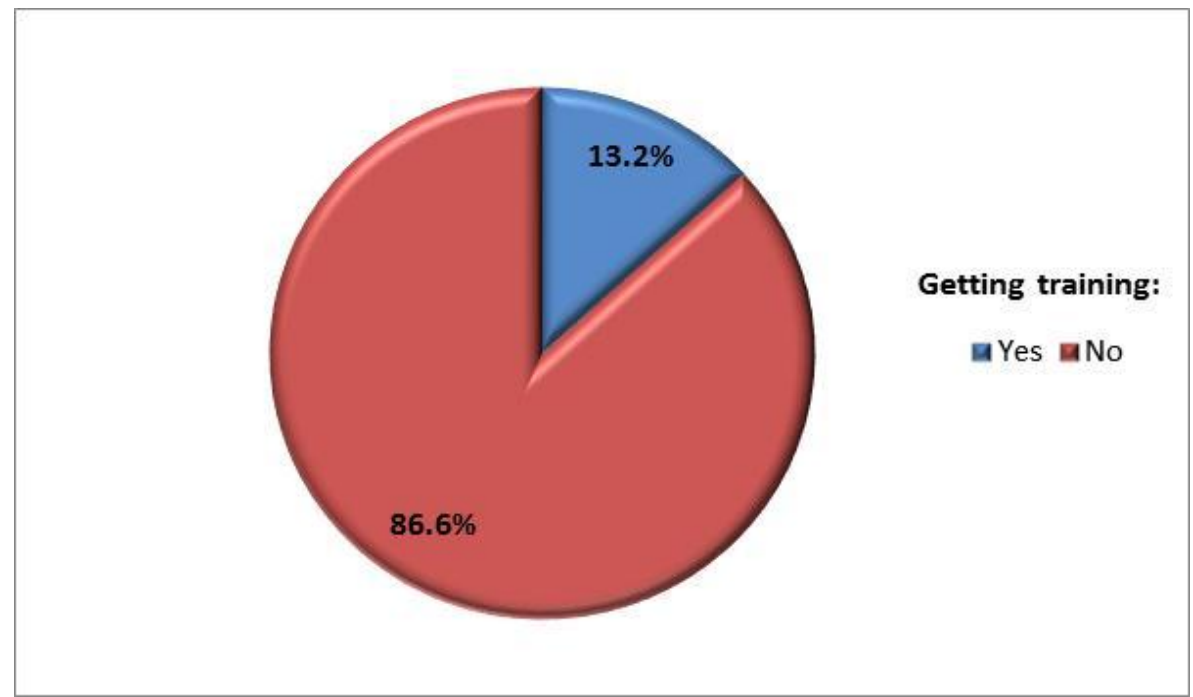

Figure (1): Percentages of getting trained on neonatal jaundice among the studied physicians

Figure (2) demonstrated the knowledge responses and score of the physicians as regards different aspects of neonatal jaundice. The knowledge score of PHC physicians was $20.4 \pm$ 3.1 which was considered as poor knowledge (less than $50 \%$ of the total score 42 ) of the physicians regarding neonatal jaundice.

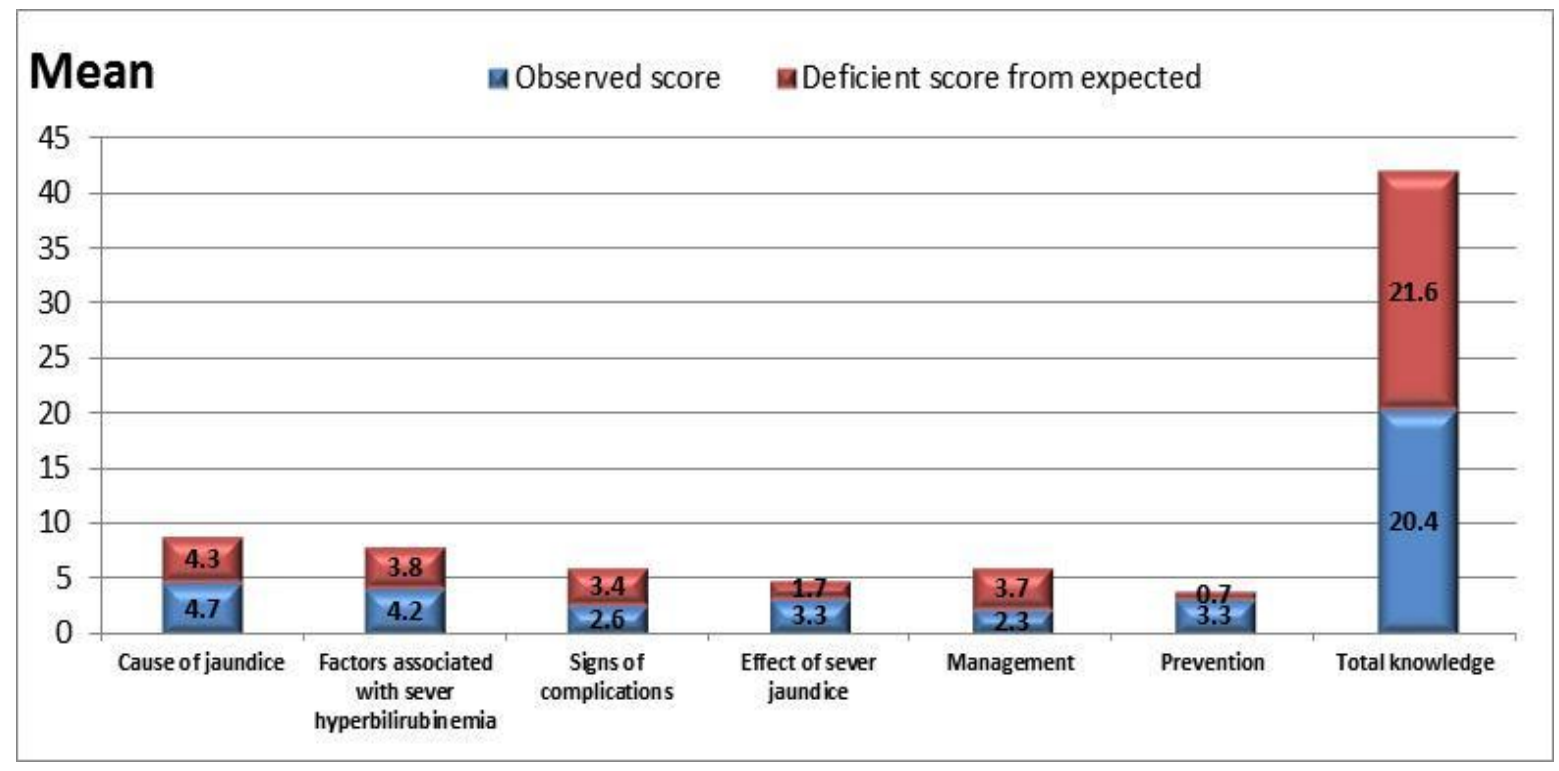

Figure (2): Deficiency in the physician's knowledge score in relation to observed score 
Figure (3) demonstrates the responses and scores of practices of the physicians as regards different aspects of neonatal jaundice. The total practices of study group regarding neonatal jaundice were generally good with a total score of $14.8 \pm 2.1$ (Expected total score 24).

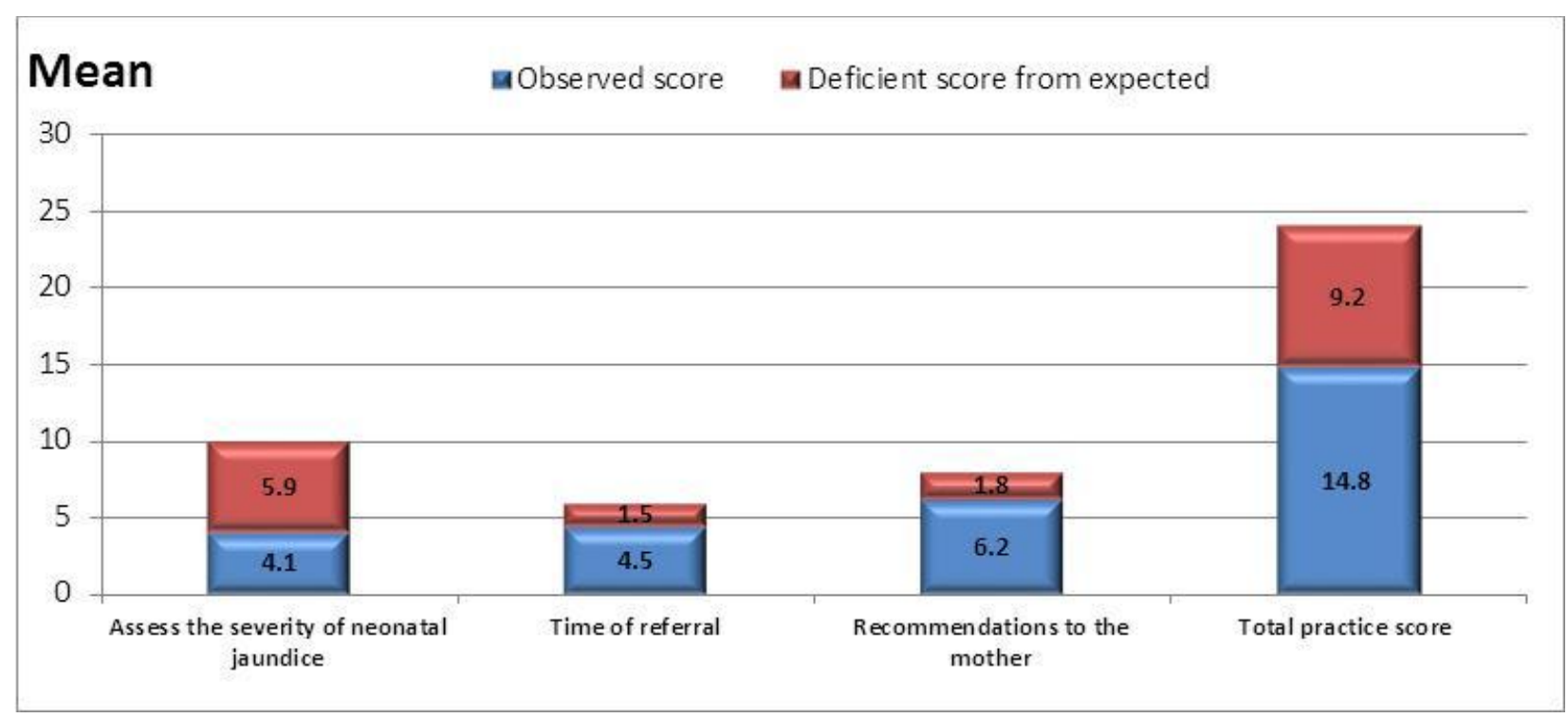

Figure (3) Practices of the study physicians on Neonatal jaundice

There was a statistically significant positive correlation between knowledge score and practice score in the studied physicians, table (1)

Table (1): Correlation of practice score with knowledge score among the physicians

\begin{tabular}{|c|c|c|}
\hline \multirow{2}{*}{ Knowledge score } & \multicolumn{2}{|c|}{ Practice score } \\
\cline { 2 - 3 } & $\mathbf{R}$ & P-value \\
\hline & 0.652 & $<0.0001 *$ \\
\hline
\end{tabular}

* Significant

The majority of study nurses $255(86.8 \%)$ had no specific training on neonatal jaundice. Also, most of the studied nurses 275 (87.3\%) reported their necessities in receiving training and information on neonatal jaundice, as demonstrated in figure (4). 


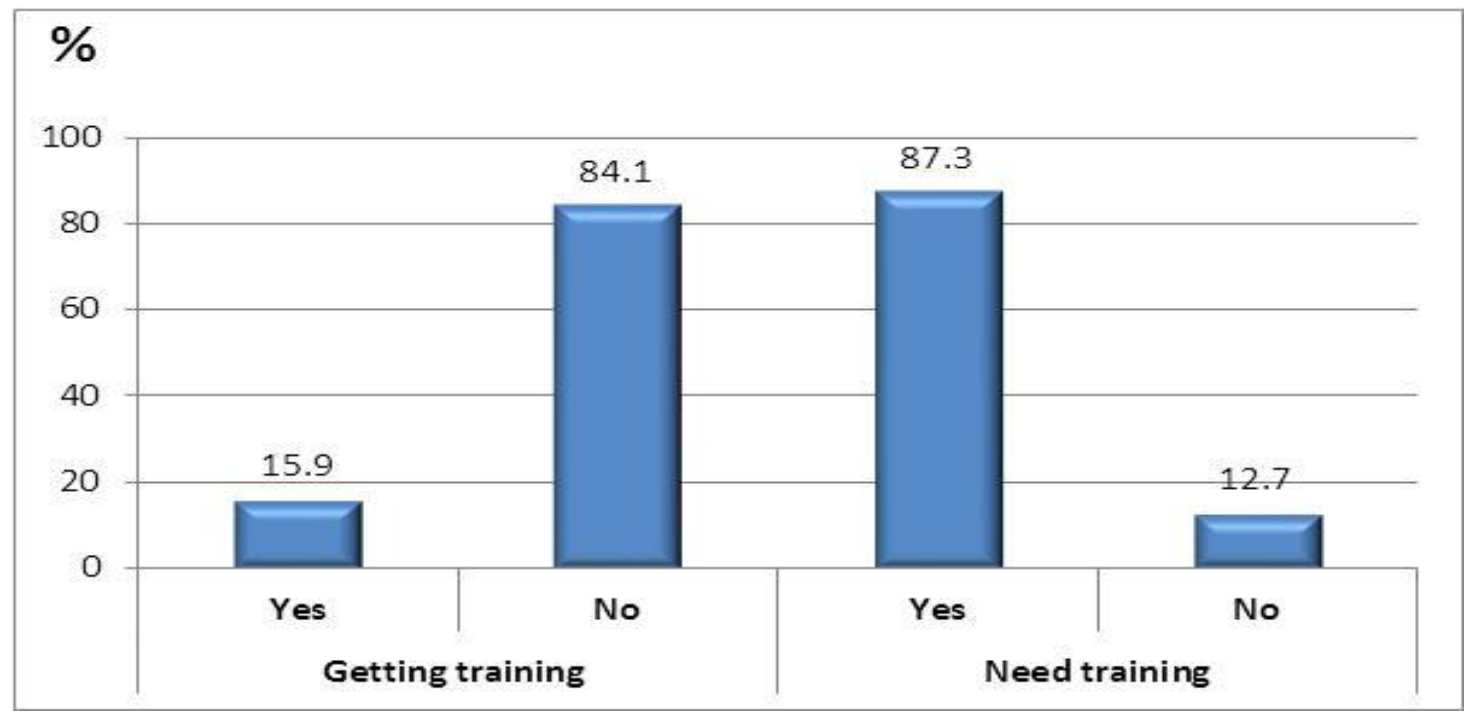

Figure (4): Training of the nurses on neonatal jaundice

Figure (5) showed the responses and scores of knowledge of the nurses about different aspects of neonatal jaundice. The total knowledge score of studied groups of nurses was 13.8 \pm 4.5 which was considered as poor knowledge (less than $50 \%$ of the total score 32 ) of the nurses regarding neonatal jaundice.

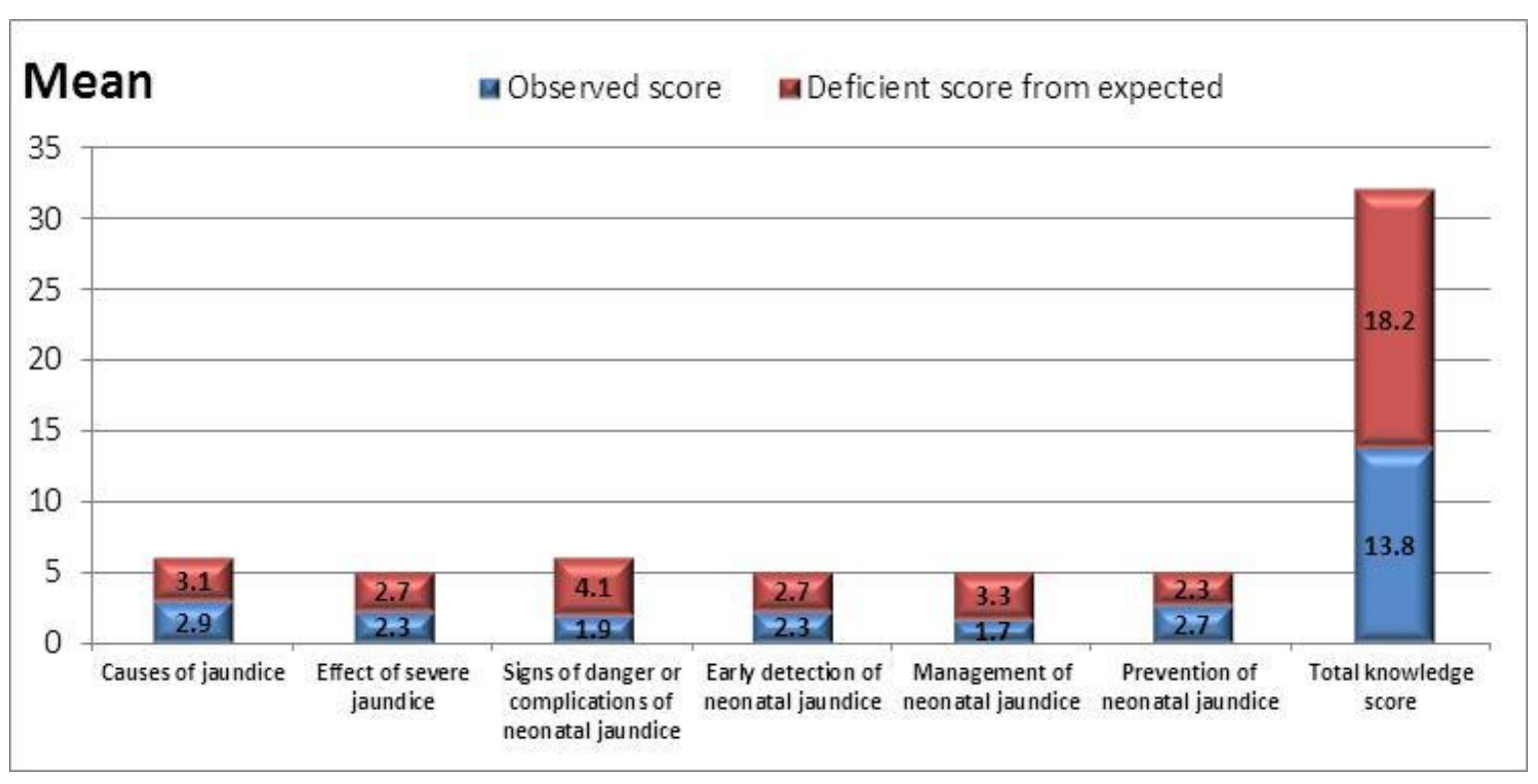

Figure (5): Knowledge score of studied nurses 
Table (2) demonstrates the responses and scores of practices of the nurses on neonatal jaundice. The total practices of study group were generally good with a total score of $7.6 \pm 1.8$

\begin{tabular}{|c|c|c|c|c|c|c|}
\hline \multirow[t]{2}{*}{ Question } & \multicolumn{2}{|c|}{ Always } & \multicolumn{2}{|c|}{ Sometimes } & \multicolumn{2}{|c|}{ Never } \\
\hline & $\mathbf{N}$ & $\%$ & $\mathbf{N}$ & $\%$ & $\mathbf{N}$ & $\%$ \\
\hline Bring baby to the Physician & 265 & 84.1 & 40 & 12.7 & 10 & 3.2 \\
\hline Referral to hospital & 190 & 60.3 & 45 & 14.3 & 80 & 25.4 \\
\hline Refer to perform S. bilirubin level & 165 & 52.4 & 95 & 30.2 & 55 & 17.5 \\
\hline $\begin{array}{l}\text { Advice mother: Expose baby to the sun } \\
\text { light }\end{array}$ & 145 & 46.0 & 70 & 22.2 & 100 & 31.7 \\
\hline $\begin{array}{l}\text { Advice mother: Expose baby to } \\
\text { fluorescence light }\end{array}$ & 80 & 25.4 & 65 & 20.6 & 170 & 54.0 \\
\hline Advice mother: did not stop breast feeding & 305 & 96.8 & 5 & 1.6 & 5 & 1.6 \\
\hline
\end{tabular}

(Expected total score 12).

\section{Table (2) Practices of study nurses on Neonatal jaundice $(\mathrm{N}=315)$}

The majority of participant nurses could do the correct practice with a percentage above 50 for all items except for two practices; advise mother to expose their babies to sun light and to fluorescence light where only $(46.0 \%$ and $25.4 \%)$ respectively of the nurses give the correct recommendations to the mothers.

There was no statistically significant correlation between knowledge score and practice score among the studied nurses, as shown in table (3).

Table (3): Correlation of practice score with knowledge score among nurses

\begin{tabular}{|c|c|c|}
\hline \multirow{2}{*}{ Knowledge score } & \multicolumn{2}{|c|}{ Practice score } \\
\cline { 2 - 3 } & $\mathbf{R}$ & P-value \\
\hline & 0.024 & 0.669 \\
\hline
\end{tabular}




\section{Discussion:}

\section{Physicians:}

In the first instance, even though almost all the physicians were aware of the condition and indeed have neonates as clients, but our study showed poor knowledge (less than 50\%) of the physicians regarding neonatal jaundice. The reason of this may be due to the majority of the studied physicians $(86.8 \%)$ had experience less than 3 years, and also lacked any specific training about neonatal jaundice. However, physicians who got training on neonatal jaundice had higher total knowledge score than those did not get any training. Similar observations of weak understanding of neonatal jaundice in terms of definitions, knowledge of causes, treatment and possible complications of severe jaundice among the health workers at primary health care levels showed by (Adebami, 2015) a study conducted at 12 local government primary health and maternity centers in Nigeria during 2014.

Ignorance of health workers based on the causes، care/treatment and complications of severe neonatal jaundice will have very serious consequences on the management of neonatal jaundice and can pose serious challenge on the reduction of bilirubin induced neurologic dysfunction in the community. So, education and training concerning jaundice should be given to update the knowledge of the primary health care workers.

Regarding causes of jaundice, in the current study most of the participant physicians mentioned the correct answers with a percentage over 50 in most of the items except for only four causes as; bacterial infection in the blood (13.2\%), breast milk jaundice $(39.5 \%)$, exaggerated Physiological jaundice (28.9\%) and subgroup incompatibility (10.5\%) mentioned the correct answer This was in agreement with results of (Gadalla, 2013) (study done in Kalubia governorate during the period from June 2011 through May 2012 on 1000 nurses and 500 physicians all were working in primary health care sector about neonatal jaundice) who reported that there was a good knowledge about causes of neonatal jaundice in doctors of primary health care units. as most of them choose commonest causes as ABO incompatibility, RH incompatibility, sepsis, prematurity and lastly physiologic jaundice. And all were ignoring writing any other causes as germ in breast or breast milk which not really a cause of jaundice.

Our study illustrated that physician's knowledge regarding measures of prevention was high. The majority of them could mention the correct answers with a percentage over 80 for all items screening for blood group and $\mathrm{RH}$ to all pregnant female, good breast feeding and good prenatal, natal \& postnatal care) except for one question; screening for TORCH infection where only $40 \%$ of them identified the correct answer. This was in agreement with (Gadalla, 2013) who mentioned that there was fairly adequate knowledge of PHC doctors in prevention 
of NNJ as a good percentage choose screening for blood group and $\mathrm{RH}$ to all pregnant female then good breast feeding and good antenatal and postnatal care and low percentage choose screening for TORCH infection.

In the current study the total practice of study group regarding neonatal jaundice was generally good with a total score of $14.8 \pm 2.1$ (Expected total score 24). Regarding assessing the severity of neonatal jaundice, they could not report sufficient practice for all items except for assessing the neonatal jaundice by total serum bilirubin assessment where all physicians did the proper practice. This was in agreement with (Gadalla, 2013) study in which a $41.3 \%$ of PHC assess the severity of NNJ by cephalocaudal evaluation. While $87.0 \%$ of PHC doctors chose to measure the level of total serum bilirubin to evaluate neonatal jaundice and its presence in neonates and only $33 \%$ of PHC physicians used transcutaneous bilirubinometer as a way to check severity of NNJ. Bilirubin measurement, either transcutaneous or in blood, is necessary to identify the infants who need treatment.

During the first days after birth, daily visits by health care workers are needed to detect the infants at risk of severe hyperbilirubinemia to check bilirubin levels (Sampurna et al., 2018).

As regards time of referral, the physicians mentioned the correct practice as refer neonates with jaundice when jaundice reaches hand and feet, and if it was prolonged. On the other hand, less than half of the physicians reported that they refer their patients in case of dark urine and pale stool. This is in agreement with (Gadalla, 2013) study in which
$60.6 \%$ of PHC doctors chose prolonged jaundice as a cause of referral to estimate bilirubin level but few of them chose dark urine, pale stool and jaundice in hand and feet. Practice of the study physicians as regard recommendations to mothers was good.

\section{Nurses:}

The total knowledge of study group regarding neonatal jaundice was generally low as the majority of the nurses $(77.8 \%)$ had experience less than 3 years, also $(86.8 \%)$ of them had no specific training on neonatal jaundice, and $(87.3 \%)$ reported their necessities in receiving training and information on neonatal jaundice.

As regards causes of neonatal jaundice, the majority of nurses could mention the correct answers in three items; breast milk jaundice, physiological jaundice and prematurity. In the other three items less than $50 \%$ of nurses identified the correct answer as bacterial infection in the blood (14.3\%), ABO incompatibility (30.2\%) and $\mathrm{RH}$ incompatibility (38.1\%) This was in agreement with (Gadalla, 2013) study concerning to the common causes of NNJ revealed another area of inadequate knowledge that $85.4 \%$ chose physiological cause, $54.8 \%$ chose prematurity and most of them neglected the other important causes and 13\% chose incorrect answer (germ in breast of breast milk). Breast feeding is an important risk factor for the development of hyperbilirubinemia, particularly if nursing is not going well (Sampurna et al., 2018).

Most of the nurses have positive perception towards jaundice and some have negative perception towards jaundice as jaundice is a disease that could be cured by treating the main cause behind the 
disease. If treated before the occurrence of complications, jaundiced patient could work as other normal individual. This perception towards NNJ should be changed through education and training program.

Conclusion: knowledge of primary health care workers about neonatal jaundice was inadequate and may cause potential delays in referral for effective treatment. There is need for regular training and re-training of primary health care workers to ensure effective management and reduce the complications of neonatal jaundice.

\section{Recommendations:}

1. The ministry of health and population should provide pre-service training to primary health care workers before taking up their jobs. And this training should include how to manage pediatric problems especially neonatal jaundice.

2. Continuous medical education programs and annual workshops should be adopted by the training department of the Health Directorate about neonatal jaundice for those working in primary health care sector as they are facing babies just after delivery at time of thyroid screening and vaccination before pediatrician see them.

\section{References:}

1. 1- Li, Y., Wu, T., Chen, L., and Zhu, Y., (2019): Associations between G6PD, OATP1B1and BLVRAvariants and susceptibility to neonatal hyperbilirubinaemia in a Chinese Han population, Journal of Pediatrics and Child Health, https://doi:10.1111/jpc.14346.

2. Olusanya, B.O., Ogunlesi, T.A., Kumar, P., Boo, N., Iskander, I.F., Almeida, M.F., Vaucher, Y.E., and
Slusher, T.M., (2015): Management of late-preterm and term infants with hyperbilirubinaemia in resourceconstrained settings, BMC Pediatrics, 15:39.

https://doi:10.1186/s12887-015-0358$\mathrm{z}$.

3. Adebami, O.J., (2015): Assessment of knowledge on causes and care of neonatal jaundice at the Nigerian primary and secondary health institutions, International Journal of Research in Medical Sciences, Vol. 3 Issue 10, Pp. 2605-2612. http://dx.doi.org/10.18203/23206012.ijrms20150799

4. Gadallah, E.F., (2013): Assessment of Knowledge and Attitude of Primary Care Providers about Neonatal Jaundice in Primary Health Care Units in Kalubia Governorate, Unpublished Master Thesis, Faculty of medicine, Benha University.

5. Sampurna, T.A., Ratnasari, K.A., Etika, R., Hulzebos, C.V., Dijk, P.H., Bos, A.F., and Sauer, J.J., (2018): Adherence to hyperbilirubinemia guidelines by midwives, general practitioners, and pediatricians in Indonesia, PLOS ONE 13(4):e0196076.

https://doi.org/10.1371/journal.pone.0 196076. 